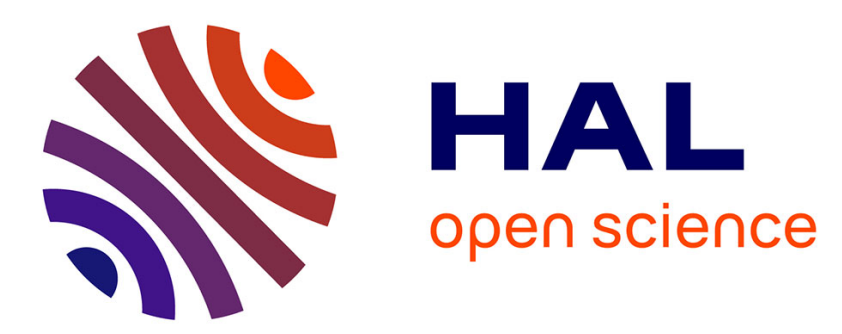

\title{
Selection of extreme events generated in Raman fiber amplifiers through spectral offset filtering
}

\author{
C. Finot, K. Hammani, Julien Fatome, J.M. Dudley, G. Millot
}

\section{To cite this version:}

C. Finot, K. Hammani, Julien Fatome, J.M. Dudley, G. Millot. Selection of extreme events generated in Raman fiber amplifiers through spectral offset filtering. IEEE Journal of Quantum Electronics, 2009, 46 (2), pp.205-213. 10.1109/JQE.2009.2030513 . hal-00456767

\section{HAL Id: hal-00456767 \\ https://hal.science/hal-00456767}

Submitted on 15 Apr 2021

HAL is a multi-disciplinary open access archive for the deposit and dissemination of scientific research documents, whether they are published or not. The documents may come from teaching and research institutions in France or abroad, or from public or private research centers.
L'archive ouverte pluridisciplinaire HAL, est destinée au dépôt et à la diffusion de documents scientifiques de niveau recherche, publiés ou non, émanant des établissements d'enseignement et de recherche français ou étrangers, des laboratoires publics ou privés. 


\title{
Selection of Extreme Events Generated in Raman Fiber Amplifiers Through Spectral Offset Filtering
}

\author{
Christophe Finot, Member, IEEE, Kamal Hammani, Student Member, IEEE, Julien Fatome,
} John M. Dudley, Senior Member, IEEE, and Guy Millot

\begin{abstract}
In this work, we discuss the emergence of rare and intense events in a Raman fiber amplifier. The temporal and spectral evolutions of continuous and pulsed signals are investigated, and approaches based on spectral slicing of the broadened spectra are proposed in order to isolate the rarest events. Numerical simulations are confirmed by experimental results.
\end{abstract}

Index Terms-Extreme events, nonlinear optics, Raman fiber amplifiers.

\section{INTRODUCTION}

$\mathbf{F}$ OR more than three decades, optical fibers have been recognized as a versatile and highly effective testbed to investigate a very wide range of nonlinear concepts, ranging from modulation instability, in scalar [1] or vectorial [2] configurations, generated by coherent [1] or incoherent [3] pumping waves, to the propagation of optical solitons [4] or similaritons [5]. More recently, it has also been demonstrated that the evolution of picosecond pulses or continuous waves in a highly nonlinear optical fiber can be characterized by "extreme value" statistics that deviate strongly from more usual Gaussian statistics [6]-[11]. A suggested analogy with hydrodynamics has led to the use of the term "optical rogue wave" as a description of such rare but intense events occurring during supercontinuum generation.

Other recent studies have shown that such extreme statistics are not restricted to soliton-supporting systems. Indeed, similar heavy tailed statistics can also be observed in other widespread fiber-based configurations such as Raman amplifiers at telecommunication wavelengths [12] or optical waveguides [13]. In fact, under the influence of the quasi-instantaneous Raman gain under conditions of low group velocity mismatch, fluctuations of a partially incoherent pump can be exponentially transferred on the amplified signal. Similar trends have also been demonstrated in parametric amplifiers driven by a

C. Finot, K. Hammani, J. Fatome, and G. Millot are with the Institut Carnot de Bourgogne, UMR 5209 CNRS-Université de Bourgogne, 21078 Dijon, France (e-mail: christophe.finot@u-bourgogne.fr, kamal.hammni@u-bourgogne.fr, julien.fatome@u-bourgogne.fr, guy.millot@u-bourgogne.fr).

J. M. Dudley is with the Institut FEMTO-ST, UMR 6174 CNRS-Universite de Franche-Comté, Besançon, France (e-mail: john.dudley@univ-fcomte.fr). partially incoherent pump [14]. In such a context, in order to study and explain the appearance dynamic of these rare events, it becomes of primary importance to find a way to control and isolate the rarest and most intense events. In the framework of supercontinuum generation, it has been demonstrated that appropriately-implemented spectral filtering by means of a off-centered optical band pass filter (OBPF) could efficiently isolate the optical rogue structures [6], [7], [10]. Basically, ultrashort pulses generated from the nonlinear dynamic fission/recompression of an initial pumping pulse are indeed strongly affected by the intrapulse Raman response of fused silica which progressively shifts their central frequency towards higher wavelengths. Consequently, recompressed pulses with the highest peak power then leads to the most frequency shifted pulses which can therefore be easily isolated.

In the context of extreme event appearance in Raman amplifiers from a continuous seed, it has been shown that the impact of cross-phase modulation (XPM) induced by a partially incoherent pump on the continuous wave leads to a spectral expansion of the amplified signal. It has also been experimentally suggested that slicing of this broadened spectrum could allow efficient isolation of these rare events [12].

In this paper, we further detail how these rare structures can be controlled in a Raman amplifier and how it is possible to discriminate the small-scale fluctuations from the giant structures. We highlight a clear distinction between the behavior affecting a continuous seed and the evolution of an initial ultrashort pulse train. The physical phenomena implied in the rare-event control can indeed significantly vary. Our paper is thus organized as follows. We first present the numerical model we developed as well as a simplified model that enables us to achieve some clear physical understanding of the underlying nonlinear physics. We then investigate the amplification of a continuous wave as well as a pulse train in the Raman amplifier. The numerical conclusions are finally compared to experimental results in the last part of our manuscript.

\section{NUMERICAL MODEL}

During its evolution in a single mode optical fiber, the slowlyvarying envelope of the electrical field $\psi(z, T)$ of an optical wave can be described by the extended nonlinear Schrödinger equation [15]:

$$
\begin{aligned}
i \frac{\partial \psi}{\partial z}=-i \frac{\alpha}{2} \psi+\frac{\beta_{2}}{2} \frac{\partial^{2} \psi}{\partial T^{2}} & +i \frac{\beta_{3}}{6} \frac{\partial^{3} \psi}{\partial T^{3}} \\
& -\gamma \psi \int_{0}^{\infty} R\left(t^{\prime}\right)\left|\psi\left(z, t-t^{\prime}\right)\right|^{2} d t^{\prime}
\end{aligned}
$$


with $\beta_{2}$ and $\beta_{3}$ describing the chromatic dispersive properties of the optical fiber, $\gamma$ the Kerr nonlinear coefficient, $\alpha$ the optical losses, $R(t)$ the silica response, which includes both the instantaneous and the delayed Raman response. $z$ and $T$ are the propagation distance and the temporal coordinate in a reference frame moving at the group velocity of the wave. $\psi(0, T)$ is the initial field made of the temporal superposition of the pump wave $\psi_{P}$ and the signal $\psi_{S}$ which is shifted by $\Omega=13 \mathrm{THz}$ with respect to the pump wavelength and therefore leads to the maximum gain response: $\psi(0, T)=\psi_{P}(0, T)+\psi_{S}(0, T) \exp (-i \Omega T)$.

As no additional frequency is generated by nonlinear frequency mixing, it is possible to isolate the evolution of the pump $\psi_{P}$ from the evolution of the signal $\psi_{S}$. We can also consider that the Raman gain experienced by the signal is constant over the spectral range under investigation $g_{R}=6.5 \times 10^{-3} \mathrm{~m}^{-1}$ and that the Raman intrapulse effect and resulting soliton self-frequency shift are not significant in the normal dispersion regime. Moreover, due to the propagation length under consideration (typically half a kilometer), the consequences of the fiber linear losses are low and can therefore be neglected. Given all these justified assumptions, (1) can be reduced to the set of two coupled nonlinear Schrödinger equations [16] shown in equation (2) at the bottom of the page, with $\delta=1 / v_{g P}-1 / v_{g S}$ the walk-off parameter defined as the difference between the group velocities $v_{g S}$ and $v_{g P}$ of the signal and the pump respectively. $\beta_{2 S}$ and $\beta_{2 P}$ are the second order dispersion of the signal and pump respectively. Note that compared to [12], we have here taken into account the effects of second order dispersion $\beta_{2}$ in order to better describe the pulsed signal dynamics. Let us also notice that compared to the widely used models for Raman amplification which rely on a power analysis [16]-[18], the model we implement here is based on the evolution of the scalar electrical field. This model will then enable us to describe the dynamics, both in the temporal and spectral domains, which is necessary in order to understand our proposed spectral filtering method.

A crucial point here is that the pump used in our study is a partially incoherent pump $\psi_{P}$ that we modeled in the spectral domain by a Gaussian intensity field having a stochastic spectral phase $\varphi(\omega)$ with a uniform distribution between $-\pi$ and $\pi$ [19], [20]:

$$
\tilde{\psi}_{P}(\omega, 0) \propto \exp \left(-2 \ln (2) \frac{\omega^{2}}{\Omega_{L}^{2}}\right) \exp (i \varphi(\omega))
$$

$\Omega_{L}$ is the spectral full-width at half maximum (FWHM) of the pump, $\Omega_{L}=17.7 \mathrm{GHz}$, which corresponds in the temporal domain to intensity fluctuations having a minimum temporal FWHM of $25 \mathrm{ps}$. We will consider an average pump power of $175 \mathrm{~mW}$, leading to an average gain of $3 \mathrm{~dB}$.
Regarding the properties of the initial signals, two kinds of signals will be investigated. First, we will study the evolution of a continuous wave with an average power of $0.5 \mathrm{~mW}$. Then, we will focus on the evolution of a Gaussian pulse train having a 2.5 ps FWHM and an initial pulse energy of $1 \mathrm{pJ}$.

Our Raman amplifier is based on a 500-meter highly nonlinear fiber, with a second order dispersion $1.3 \times 10^{-3} \mathrm{ps}^{2} / \mathrm{m}^{-1}$ and $2.2 \times 10^{-3} \mathrm{ps}^{2} / \mathrm{m}^{-1}$ at signal and pump wavelengths respectively. The nonlinearity is $\gamma=10 \mathrm{~W}^{-1} \cdot \mathrm{km}^{-1}$ and the walk-off coefficient is $\delta=0.10 \mathrm{ps} \cdot \mathrm{m}^{-1}$, which leads to an integrated walk-off of 50 ps between the pump and the signal. Our fiber is normally dispersive, which enables us to ignore modulation instability [1] as well as solitonic recompression [4].

\section{Numerical Results For a CONTINUOUS SEED}

We first investigate the evolution of a continuous signal. We have outlined in a previous work [12] that a continuous signal evolving in a Raman fiber amplifier exhibiting low walk-off between pump and signal will experience an incoherence transfer of noise from the pump to the signal leading to the generation of rare and intense spikes.

\section{A. Simulations Based on a Simplified Model}

In order to better understand the physics behind the origin of the formation of those spikes, we propose to consider as a first qualitative approach a simplified model. In this approach, the pump, amplifying the considered continuous seed, is reduced to a single Gaussian pulse with a temporal width of 25 ps corresponding to the shortest temporal width possible in the incoherent pump structure.

We have plotted in Fig. 1(a) the initial pump (a1) and signal (a2) as well as the resulting amplified output signal (a3). As expected, the continuous seed has been amplified and a replica of the pump profile can be observed on the output signal (Fig. 1(a3) plotted on a semi-logarithmic scale). Fig. 1(b) outlines that the amplification undergone by the signal varies exponentially with the pump peak-power, confirming that the process is not affected by any depletion. Simply stated, we can expect any fluctuation of the initial pump to be exponentially transferred on the signal. It should be also noted that due to the walk-off value which is superior to the finite temporal width of the pump pulse, the amplification is much lower than the value in absence of walk-off: $\exp \left(g_{R} L \psi_{P}^{2}\right)$ [21].

If we now get concerned with the spectral intensity profile of the amplified signal (see Fig. 1(c)), we can note a significant spectral expansion of the initial continuous signal. Such a broadening is caused by the XPM of the pump on the signal [22], [23]. It is then possible to slice the broadened spectrum by using a frequency offset OBPF in order to remove the central

$$
\left\{\begin{array}{l}
\frac{\partial \psi_{P}}{\partial z}=-\frac{g_{R}}{2}\left|\psi_{S}\right|^{2} \psi_{P}-i \frac{\beta_{2 P}}{2} \frac{\partial^{2} \psi_{P}}{\partial T^{2}}+i \gamma\left[\left|\psi_{P}\right|^{2}+2\left|\psi_{S}\right|^{2}\right] \psi_{P} \\
\frac{\partial \psi_{S}}{\partial z}=\frac{g_{R}}{2}\left|\psi_{P}\right|^{2} \psi_{S}-i \frac{\beta_{2 S}}{2} \frac{\partial^{2} \psi_{S}}{\partial T^{2}}+i \gamma\left[\left|\psi_{S}\right|^{2}+2\left|\psi_{P}\right|^{2}\right] \psi_{S}+\delta \frac{\partial \psi_{S}}{\partial T}
\end{array}\right.
$$



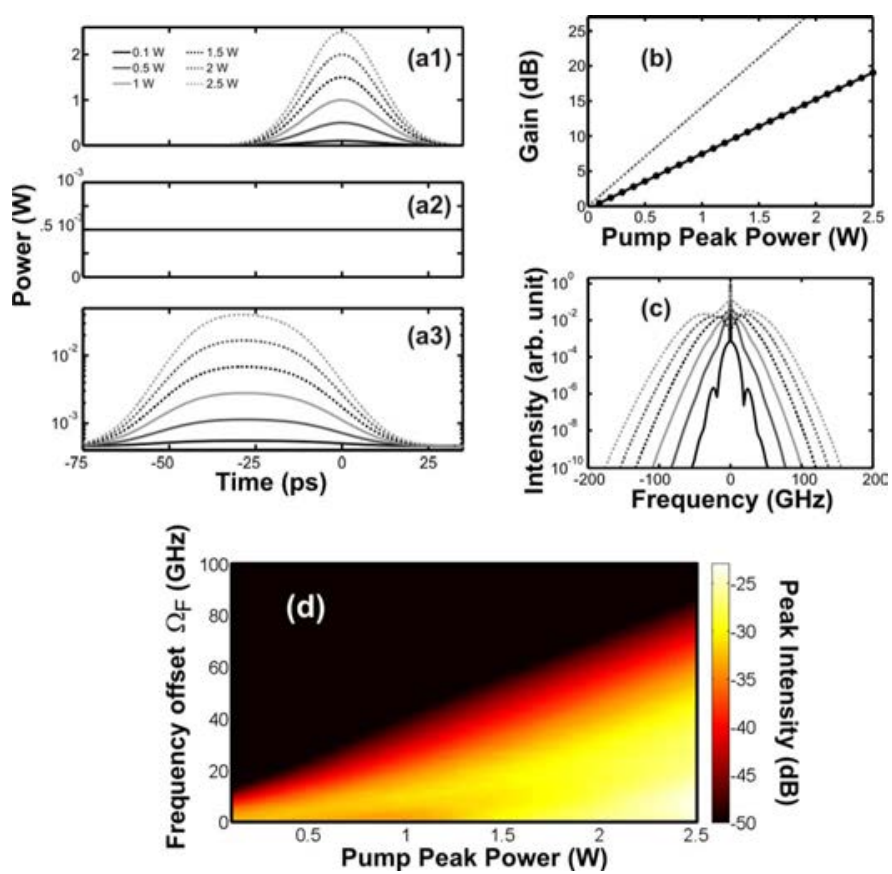

Fig. 1. (a) Temporal intensity profiles of the initial pump (a1) and continuous signal (a2). The signal after amplification is plotted on a semilogarithmic scale (a3). (b) Intensity evolution of the gain (defined as the ratio of the output peak power of the signal and the initial average signal power) obtained from our numerical simulation (black dotted-line) and predicted from the formula $\exp \left(g_{R} L \psi_{P}^{2}\right)$ (dashed-line) (c) Ouput signal spectrum for various pump powers (same convention as Figs. a). (d) Transfer function describing the output signal peak power for various pump powers and various frequency offset filterings.

continuous part of the signal [24]. The principle of such a technique has already been successfully demonstrated in the field of wavelength conversion of high bit-rate optical telecommunication signals [25]: the intensity information of the most powerful wave (the pump) is converted on the continuous seed via XPM.

Peak powers of pump and sliced signal are therefore linked via a function that is usually called transfer function. The shape of this function depends critically on the spectral offset $\Omega_{F}$ of the OBPF. In order to illustrate this point, we have used a Gaussian filter with a spectral FWHM of $9 \mathrm{GHz}$. We can see from Fig. 1(d) that for higher detuning values $\Omega_{F}$, it becomes possible to efficiently reject the low pump powers: the frequency filter behaves as an efficient intensity discriminator whose threshold can be continuously tuned according to $\Omega_{F}$.

Let us point out that previous experimental configurations have also taken advantage of Raman amplification [25], [26]. But using an external counterpropagating Raman pump enables them to avoid any influence of this additional wave on the expected wavelength conversion process. Our scheme here is more complex as the copropagating Raman pump and the XPM pump are intrinsically a single wave and consequently induce simultaneously correlated intensity and phase changes on the signal.

We can also compare our approach to spectral selection process used in the framework of supercontinuum generation [6], [7], [10], which essentially relies on the intensity-dependant self-frequency shift undergone by ultrashort soliton pulses. In this context, spectral filtering is only possible at longer wavelength edge and the associated frequency shift is usually of the order of several tens of THz. In our approach, as the spectral expansion of the amplified signal induced by XPM is close from being symmetric (Fig. 1(c)) and does not rely on the intrapulse Raman response, filtering is possible both at shorter and longer wavelengths [12]. However, a drawback is that a significant part of the signal structure is lost during the filtering process.

\section{B. Simulations Based on an Initial Incoherent Wave}

In order to check the validity of the qualitative conclusions obtained based on our previous simplified model, we have run intensive numerical simulations based on a partially incoherent pump as described by (3). Fig. 2(a1) illustrates the initial pump intensity profile plotted on a $50 \mathrm{~ns}$ range. We have also computed the statistical distribution of the peak-powers present in this pump (calculating these statistics required a temporal window of $20 \mu \mathrm{s})$. The results plotted in Fig. 2(b1) illustrate that peak powers deviating significantly from the average pump power can exist. It is indeed possible to observe a structure having a peak-power more than 15 times the average power (which justifies a posteriori the study in part A of Gaussian pulses with initial peak powers as high as $2.5 \mathrm{~W}$ ). Compared to previous studies dealing with fluctuations of a few tens of percents of the peak-power of pulses delivered by nanosecond $Q$-switched laser pumps [13], [18], [27], [28], the partially incoherent pump under investigation shows here much larger deviations.

The amplified signal (Fig. 2(a2)) exhibits significant peaks resulting from the instantaneous exponential amplification of the pump initial fluctuations. As expected from our simplified model, we can see from this figure as well as from Fig. 2(b2) that the fluctuation amplitude in the amplified signal is much larger than those present in the initial pump. From the $2 \times 10^{5}$ peaks recorded on a temporal window of $20 \mu$ s, several giant peaks have been found to have peak intensity more than 100 times the average value of the signal. We also note that the probability distribution obtained for the amplified signal (Fig. 2(b2)) is in clear agreement with a linear function when plotted on a log-log scale, consistent with the characteristic power law nature of extreme events. We can also compare the resulting distribution to the distribution one can expect if we consider the incoherent pump as a succession of isolated 25-ps Gaussian identical pulses (such as the simplified model developed in part IIIA) having a peak power distribution following the results of Fig. 2(b1) and an instantaneous gain as Fig. 1(b). We see that this model is qualitatively correct in retrieving a heavily tailed distribution, but fails in quantitatively predicting the values of the maximum peak powers that can be achieved: our simplified model underestimates the peak power value of the giant structures. This is linked to the fact that in this model, we consider a single isolated pump pulse of fixed temporal width whereas in the initial incoherent wave, due to the walk-off effect, the signal can be subject to amplification by several adjacent substructures, which increases the resulting amplification.

The spectrum of the amplified wave (Fig. 2(c)) also exhibits a noticeable reshaping. As discussed above, the initial Dirac spectrum has been significantly broadened under the effect of XPM of the pump on the signal [23], [29], [30]. Finally, we have 

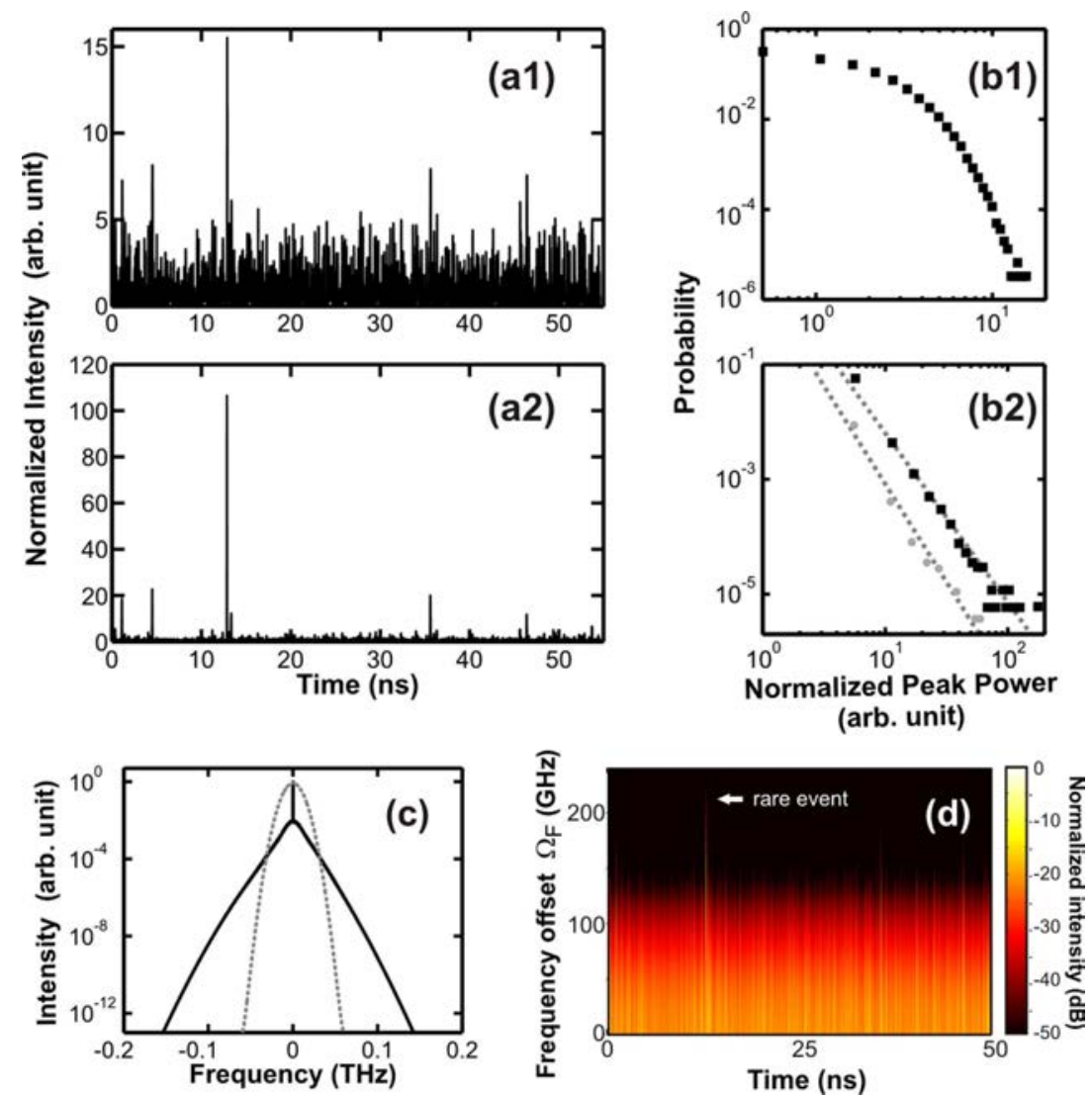

Fig. 2. (a) Initial pump intensity profile (a1) and probability of the peak powers (black squares) normalized to the average power of the pump (b1) compared with the amplified signal properties (a2 and b2). Distribution obtained with numerical simulations is compared with the distribution obtained from the initial power pump distribution and the gain derived from part 3B (grey circles). Statistical distributions plotted on a log-log scale are compared with a linear fits (grey dotted lines) (c) Output spectrum of the amplified signal (solid line) compared with the initial pump spectrum (grey dotted line) (d) Evolution of the temporal intensity profile of the signal after filtering by an offset OBPF for various offset values $\Omega_{F}$.

checked that offset spectral filtering enables us to efficiently isolate the highest peak-power structures. As shown in Fig. 2(d), higher frequency detunings lead to higher intensity discrimination: it is therefore possible to isolate a single rare event generated by the partially incoherent pump.

\section{Numerical Results Based on a Pulsed Signal}

Let us now investigate the evolution of an initial pulsed signal with a temporal width below the coherence time of the pump.

\section{A. Simplified Model}

As in the previous part, we can first study a simplified approach to obtain some physical insights about the pump to signal interactions. In the context of a pulsed signal, it is necessary to take into account an additional parameter, namely, the initial temporal delay (noted $\Delta T$ ) between the pump pulse and the signal pulse. Indeed, if in the context of a continuous seed, maximum of the amplified pulse and pump were synchronized, in the context of pulsed signal, this is not a priori always the case. The impact of this initial delay $\Delta T$ can be seen in Fig. 3(a) where we have plotted the amplified signal for different values of $\Delta T$. From our simulations, we have made out that an initial delay of $\Delta T=38$ ps leads to the rise of higher peak powers. In other words, if in the continuous wave, the pump amplitude was the single parameter influencing the amplitude of the resulting pulse, in the pulsed case, we will have two parameters that will influence the final state: the amplitude of the initial state and the temporal delay between the pump and signal.

In Fig. 3(b), we have plotted the temporal intensity profiles of the initial and final pump and signal. The temporal intensity profile of the pulse propagating in the normally dispersive amplifier progressively evolves towards a parabolic state [5], [31]. Some temporal asymmetry can however be seen due to the temporal walk-off and the depletion effects of the pump during the propagation [32]. The consequences of the strong temporal depletion experienced by the pump (Fig. 3(b)) [33] can also be seen on the gain evolution according to the pump power plotted in Fig. 3(c) which deviates from the usual linear trends and we can anticipate that the statistical properties of the amplifier will be impacted [34]. Note that compared to the previous part, we are much more affected by depletion effects here because the initial peak power is much higher than in the continuous case (we have here an initial peak power of $175 \mathrm{~mW}$ instead of $0.5 \mathrm{~mW}$ ). Furthermore, we stress that some negative gain could be observed, which is mainly due to the dispersion-induced temporal broadening of the signal pulse which consequently undergoes a peak power reduction.

Regarding the optical spectrum, noticeable broadening can be noticed as well as some spectral asymmetry which is a consequence of the pump-signal walk-off [33], [35]. We have numer- 

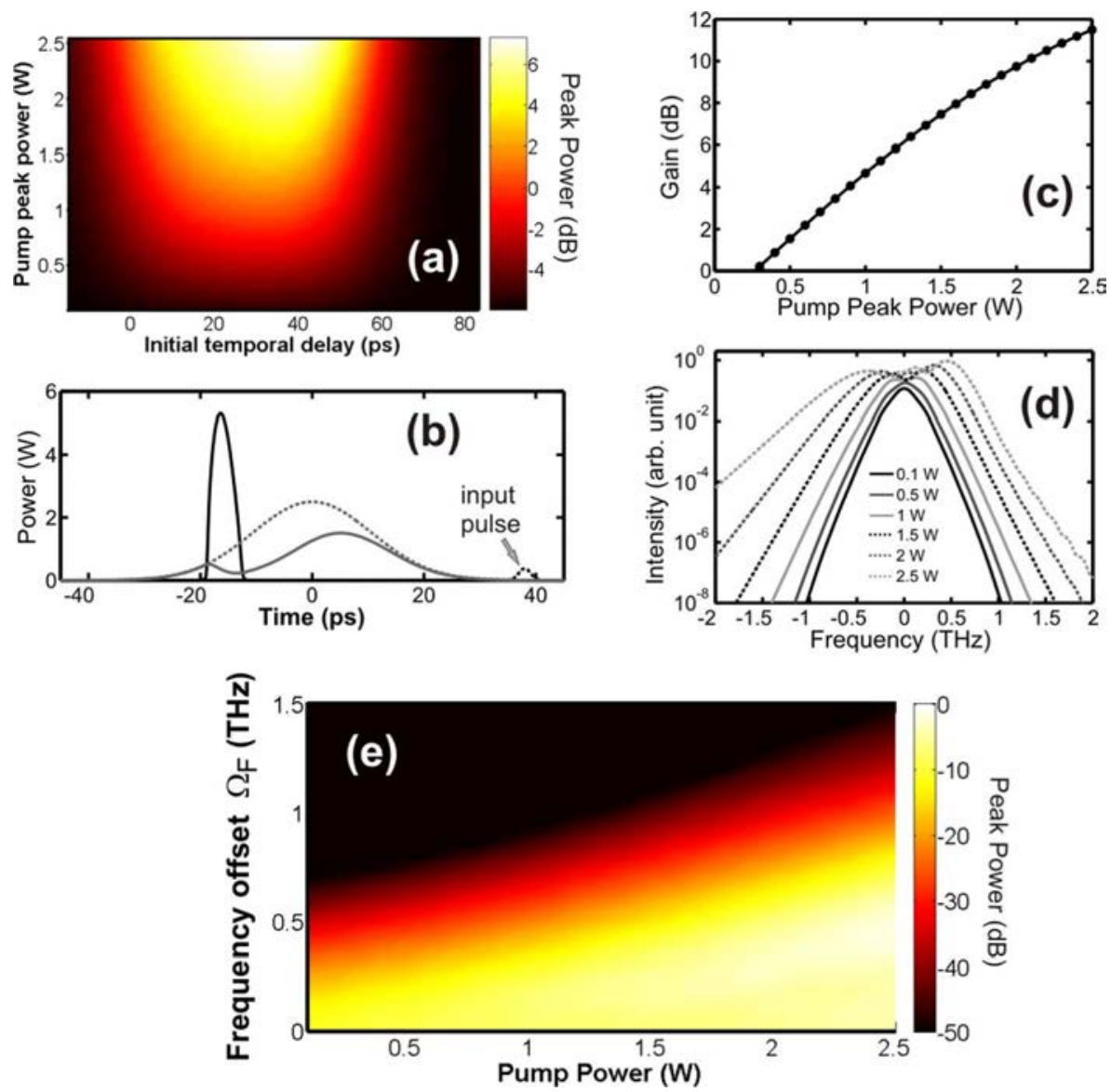

Fig. 3. (a) Output peak power of the amplified pulse for various initial pump peak powers and various initial delays. (b) Temporal intensity profiles of the signal (black) and the pump (grey). Output profiles (solid lines) are compared with initial profiles (dotted lines). Results obtained for the optimum initial temporal delay and a pump peak power of $2.5 \mathrm{~W}$. (c) Evolution of the gain versus pump peak power (gain defined as the ratio between the final and initial pulse peak power) for the optimal initial temporal delay. (d) Evolution of the output spectral intensity profile of the pump for various pump powers (results at the optimum initial temporal delay). (e) Transfer function describing the output signal peak power for various pump powers and various values of frequency offset $\Omega_{F}$.

ically checked that the spectral expansion originates not from cross phase modulation but rather from self-phase modulation (SPM) [15]. Note that the optical broadened spectra here are much larger those obtained from a continuous wave, because of a broader initial spectrum and also because the effects of SPM cannot be neglected with the much higher initial peak power

As in the preceding part, we can use an offset OBPF to isolate the structures with the highest peak powers. We have therefore plotted in Fig. 3(e) the transfer function obtained for the optimum delay $\Delta T$ for various values of the spectral offset of a Gaussian OBPF having a spectral FWHM of $100 \mathrm{GHz}$. Similarly to the previous case, increasing the spectral offset $\Omega_{F}$ can continuously change the threshold of the transfer function. Such a method based on SPM in an amplifier has already been demonstrated in the telecommunication field with a continuous Raman pumping [36]. It can also be seen as an extension of the well-known signal regeneration technique proposed by Mamyshev [37], [38] and the fact that the extinction level increases with the offset is fully consistent with the previous theoretical analysis [39].

\section{B. Simulations With an Initial Incoherent Wave}

In order to confirm our qualitative results, we have performed simulations including an incoherent pump such as described by
(3). We have run more than 12000 simulations and the resulting shot-to-shot statistics of the peak powers, plotted in Fig. 4(a), black squares, using a log-log scale, exhibits the linear tail characteristic of extreme phenomena.

We have also plotted in Fig. 4(b) the spectrogram of the output amplified signal (i.e., a spectro-temporal representation) where a pronounced event with a behavior deviating strongly from the average behavior can be noticed. The presence of this extreme shot confirms that pulses amplified to the highest peak power also exhibit the most broadened spectrum.

By recording the number of pulses after spectral slicing, we obtain the resulting statistics described in Fig. 4(c), which clearly reflects that for a low spectral offset (below $0.4 \mathrm{THz}$ ), the proportion of pulses recovered after slicing is constant to 1. A statistical analysis of the peak powers of the filtered pulse train (Fig. 4(a), grey diamonds) reveals that filtering at the central frequency behaves as a beneficial instantaneous optical power limiter [40], [41]. Indeed, the peak power fluctuations have been divided by more than 2 and we can also make out a statistical distribution reshaping. For further increase of the spectral offset $\Omega_{F}$, the number of pulses recovered after spectral filtering drops rapidly down to a very low value. We have finally found empirically that the rate of this decrease versus the frequency offset could be well approximated by an 

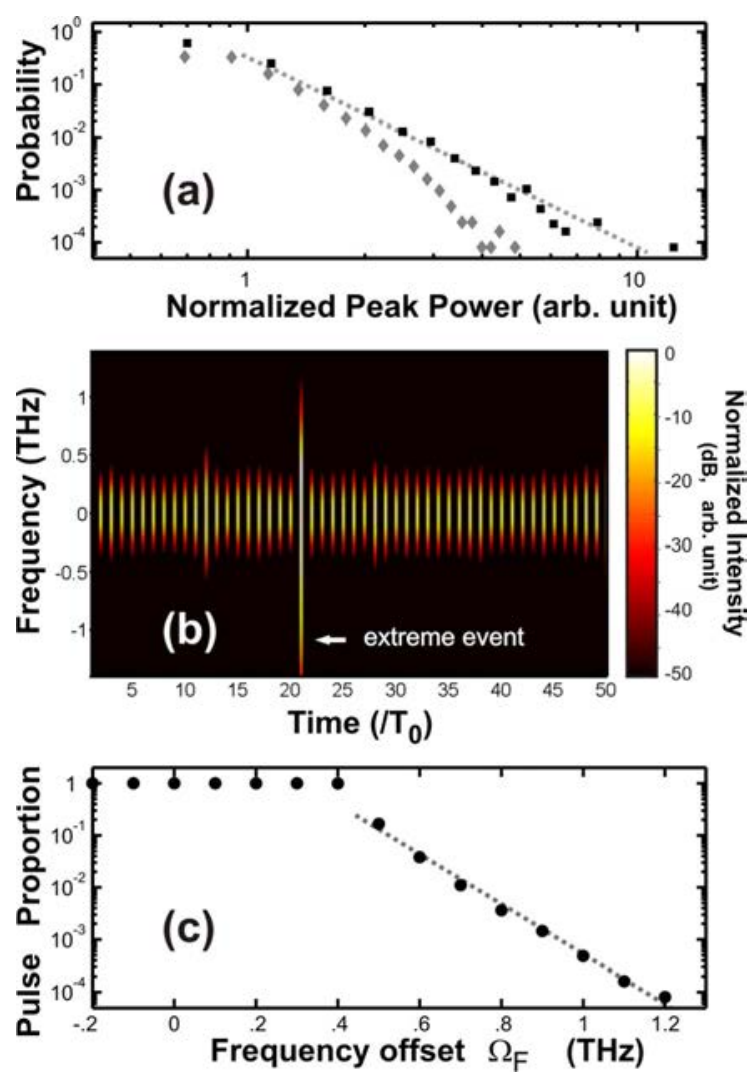

Fig. 4. (a) Statistical distribution of the output peak-power probability (black squares) (35 equidistant bins have been used). The tail of the distribution is compared to a linear function (on a log-log scale). Distribution obtained after spectral filtering at $\Omega_{F}=0 \mathrm{GHz}$ is plotted with grey diamonds (b) Time-Frequency view of an output pulse sequence of 50 pulses $\left(T_{0}\right.$ is the period of the mode locked train.) (c) Proportion of output pulses (defined as the ratio between the number of pulses detected after spectral filtering and the initial number of pulses) according to the spectral offset $\Omega_{F}$. Results for $\Omega_{F}>0.4 \mathrm{THz}$ are compared to a linear curve (using a semilog scale).

exponential decreasing law (a linear function when plotted on a semi logarithmic scale).

\section{EXPERIMENTAL VALIDATION}

\section{A. Experimental setup}

In order to confirm our numerical model, we have implemented the experimental setup illustrated in Fig. 5(a). The Raman pump is delivered by a fiber Raman laser with an initial average power of $350 \mathrm{~mW}$ at an initial wavelength of $1455 \mathrm{~nm}$. As the pump is not polarized, this average pump power of $350 \mathrm{~mW}$ corresponds in our scalar numerical model to a value of $175 \mathrm{~mW}$ [16]. Such a power experimentally leads to an average experimental gain of $3 \mathrm{~dB}$. With such reduced pump power and gain, we have not observed any spontaneous Raman emission so that we can neglect this effect in the modeling. The autocorrelation function of the pump wave is plotted in Fig. 5(b), grey solid line, and highlights a typical contrast of $1: 2$ with a coherence time of $25 \mathrm{ps}$ [12], [19], with a shape typical of the Gaussian distribution (circles) assumed for our numerical simulations (3).

The polarized signal is launched into a commercially available highly nonlinear fiber (HNLF) from ofs with parameters

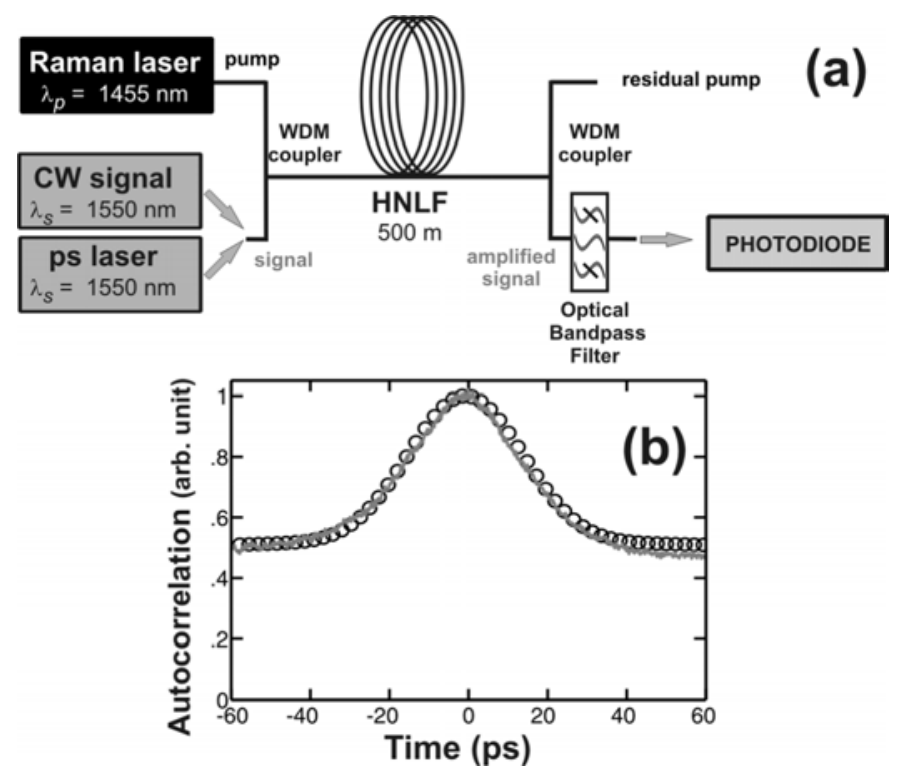

Fig. 5. (a) Experimental setup. (b) Experimental autocorrelation function of the pump wave (grey solid line) compared with the autocorrelation obtained based on the assumption of Gaussian fluctuations (circles) (3).

described in Section I. We will test the two kinds of signals. The initial continuous wave signal $(\mathrm{CW})$ is delivered around $1550 \mathrm{~nm}$ by means of an external cavity laser having a spectral linewidth below a few MHz. Regarding the pulsed signal, it is delivered by a 2.5 -ps mode-locked Erbium doped fiber laser running at a repetition rate of $22 \mathrm{MHz}$. Both signal and Raman pump are coupled into the HNLF by means of a WDM coupler. At the output of the optical fiber, a second WDM coupler enables us to select the amplified signal and remove the residual pump. Previous experiments reported in [12] have demonstrated that the extreme statistics generated by this system is in qualitative agreement with numerical predictions resulting from the model described in Section II. In the experimental section of this article, we focus our attention more specifically on the spectral selection process that can be performed by spectral filtering. In the case of the continuous seed, we have used a fiber Bragg grating having a spectral FWHM of $9 \mathrm{GHz}$. In the case of the pulsed signal, we have inserted a wavelength demultiplexer with eight output channels having each a spectral FWHM of $100 \mathrm{GHz}$ and spaced by $200 \mathrm{GHz}$. At the output of the system, the signal is finally analyzed by means of a photodiode.

\section{B. Experimental Selection of the Extreme Events}

Experimental results obtained in the case of a continuous seed with an initial power of $0.5 \mathrm{~mW}$ are plotted in Fig. 6(a). As the position of the central frequency of the fiber Bragg grating is fixed, the filter-signal frequency detuning $\Omega_{F}$ is obtained by simply adjusting the wavelength of our initial $\mathrm{CW}$ signal. It is readily apparent from subplots a1 to a3, that higher the signalfilter detuning is, lower the number of observed spikes is. As expected, for the largest detuning, only the most intense spikes are conserved.

We have also carried out some similar experiments for the 22-MHz picosecond pulsed seed. Initial pulses have a FWHM of $2.5 \mathrm{ps}$ for an average power of $-16 \mathrm{dBm}$ ( $1 \mathrm{pJ}$ energy per pulse). 

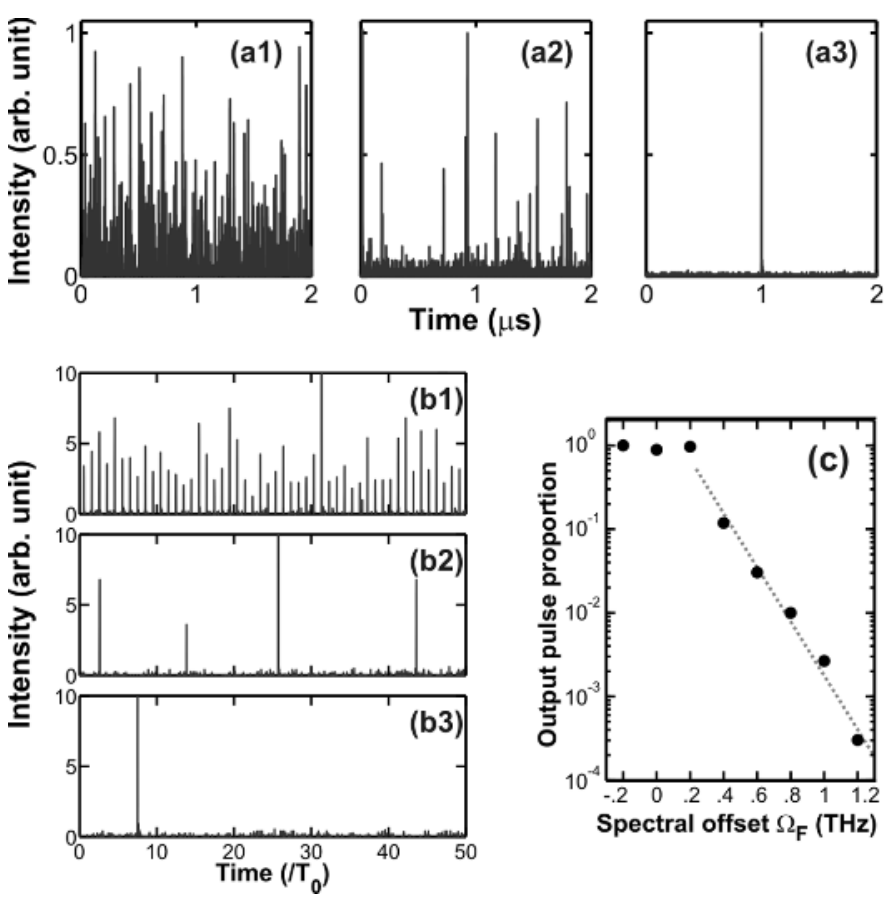

Fig. 6. (a) Experimental temporal results obtained for a initial continuous wave for different filter-signal spectral detunings: offset of $0 \mathrm{GHz}, 100 \mathrm{GHz}$ and 200 $\mathrm{GHz}$, subplots a1, a2 and a3, respectively. (b) Experimental temporal trace (temporal scale is normalized by the repetition rate) of the output amplified $22-\mathrm{MHz}$ pulsed train obtained for various filter-signal frequency detunings: $0 \mathrm{GHz}, 600$ $\mathrm{GHz}$ and $1 \mathrm{THz}$, subplots b1, b2 and b3, respectively (c) Number of detected output pulses as a function of the spectral detuning imposed by the wavelength demultiplexer. Results are compared with a linear decreasing function (using a semilog scale).

Results are recorded by means of a photodiode and an oscilloscope having a $1-\mathrm{GHz}$ electrical bandwidth so that the experimental observed spikes are mainly correlated to the pulse energy rather than peak-power. Nevertheless, recent studies have shown that statistics of peak-power or energy exhibit similar behavior [10].

If the pulse train is filtered at its corresponding central frequency, we can retrieve after amplification and filtering a pulse train at the initial repetition rate, Fig. 6(b1). However, a high amplitude jitter is clearly visible. Now, if we analyze the output signal at another port of the demultiplexer, corresponding to a higher frequency offset (Figs. b2 and b3), we can observe a dramatic drop in the number of resulting output pulses. The output signal does not present any periodicity, so we have therefore generated a pulse train having a stochastic pulse separation (with a time-spacing corresponding to a multiple of the initial pulse train period). We note that compared to the method developed in [42] and [43], which was also based on the properties of sliced broadened spectra, our resulting pulse train presents here noticeable intensity fluctuations.

Finally, we have experimentally measured the number of output pulses as a function of the initial offset (Fig. 6(c)). In good agreement with results presented in Fig. 6(b), for low filter-signal frequency detuning $(<200 \mathrm{GHz})$, the number of detected pulses corresponds to the initial number of pulses. For higher detunings, we have recorded an exponential drop of the number of intensity spikes after spectral filtering. This result is fully consistent with our numerical study developed in Section IV. We can qualitatively see that it is possible to isolate and detect by this technique a pulse having a very low probability of occurrence (around 1 over 1000).

\section{CONCLUSION}

In this work, we have proposed a simple model that has enabled us to isolate and characterize the nonlinear dynamics of the temporal and spectral evolution originating from the rare events with a non-Gaussian probability distribution. Based on the evolution of a Gaussian pulse having a temporal width corresponding to the typical coherence time scale of the incoherent wave, it becomes possible to easily understand the physical process stimulating the rogue events. Depending on the nature and power of the initial seed signal (depending if the initial pulse temporal width is below or above the coherence time), there will be a predominating role of the cross or self phase modulations respectively.

Even for a gain as low as $3 \mathrm{~dB}$, resulting fluctuations can be significant, which requires to take into account this effect for the practical design of amplifiers based on quasi-instantaneous gain: large amplitude fluctuations of the partially incoherent pump can therefore lead to large signal distortions and errors under certain conditions. In this context, we believe that our characterization and interpretation of the noise properties in the picosecond temporal domain could be useful to explore further aspects of extreme value fluctuations occurring in optical fiber propagation. Our numerical analysis has been confirmed by experimental results Our conclusions could also complement the well-known RIN measurements carried out in the electrical spectral domain to characterize amplifier noise properties [16], [2], [34], [44]-[46]. Given the close links with the experimental techniques reported here and current approaches to optical regeneration, our results may also impact on the design and implementation of future-generation transmission networks.

\section{ACKNOWLEDGMENT}

The authors would like to thank A. Picozzi and S. Pitois for illuminating discussions.

\section{REFERENCES}

[1] K. Tai, A. Hasegawa, and A. Tomita, "Observation of modulational instability in optical fibers," Phys. Rev. Lett., vol. 56, pp. 135-138, 1986.

[2] E. Seve, P. Tchofo-Dinda, G. Millot, M. Remoissenet, J. M. Bilbault, and M. Haelterman, "Modulational instability and critical regime in a highly birefringent fiber," Phys. Rev. A, vol. 54, pp. 3519-3533, 1996.

[3] A. Sauter, S. Pitois, G. Millot, and A. Picozzi, "Incoherent modulation instability in instantaneous nonlinear Kerr media," Opt. Lett., vol. 30, pp. 2143-2145, 2005.

[4] L. F. Mollenauer, R. H. Stolen, and J. P. Gordon, "Experimental observation of picosecond pulse narrowing and solitons in optical fibers," Phys. Rev. Lett., vol. 45, pp. 1095-1098, 1980.

[5] J. M. Dudley, C. Finot, G. Millot, and D. J. Richardson, "Self-similarity in ultrafast nonlinear optics," Nat. Phys., vol. 3, pp. 597-603, 2007.

[6] D. R. Solli, C. Ropers, P. Koonath, and B. Jalali, "Optical rogue waves," Nature, vol. 450, p. 1054, 2007.

[7] J. M. Dudley, G. Genty, and B. J. Eggleton, "Harnessing and control of optical rogue waves in supercontinuum generation," Opt. Express, vol. 16, pp. 3644-3651, 2008. 
[8] B. Kibler, C. Finot, and J. M. Dudley, "Soliton and rogue wave statistics in supercontinuum generation in photonic crystal fibre with two zero dispersion wavelengths," Eur. Phys. J. Special Topics, vol. 173, pp. 273-279, 2009.

[9] D. R. Solli, C. Ropers, and B. Jalali, "Active control of rogue waves for stimulated supercontinuum generation," Phys. Rev. Lett., vol. 101, pp. 233902-233902, 2008.

[10] C. Lafargue, J. Bolger, G. Genty, F. Dias, J. M. Dudley, and B. J. Eggleton, "Direct detection of optical rogue wave energy statistics in supercontinuum generation," Electron. Lett., vol. 45, pp. 217-219, 2008.

[11] A. Mussot, A. Kudlinski, M. I. Kolobov, E. Louvergneaux, and M. Taki, "Convective instabilities and optical rogue waves in fibers with CW pumping," in LEOS Winter Topical Meeting, Innsbruck, 2009, pp. TUC2.5-TUC2.5.

[12] K. Hammani, C. Finot, J. M. Dudley, and G. Millot, "Optical roguewave fluctuations in fiber Raman amplifiers," Opt. Express, vol. 16, pp. 16467-16474, 2008.

[13] D. Borlaug, S. Fathpour, and B. Jalali, "Extreme value statistics in silicon photonics," IEEE Photon. J., vol. 1, pp. 33-39, 2008.

[14] K. Hammani, C. Finot, and G. Millot, "Emergence of extreme events in fiber-based parametric processes driven by a partially incoherent wave," Opt. Lett., vol. 34, pp. 1138-1140, 2009.

[15] G. P. Agrawal, Nonlinear Fiber Optics, Third ed. San Francisco, CA: Academic Press, 2001.

[16] C. Headley and G. P. Agrawal, Raman Amplification in Fiber Optical Communications. : Academic Press, 2005.

[17] J. Bromage, "Raman amplification for fiber communications systems," J. Lightw. Technol., vol. 22, pp. 79-93, 2004.

[18] A. Betlej, P. Schmitt, P. Sidereas, R. Tracy, C. G. Goedde, and J. R. Thompson, "Increased stokes pulse energy variation from amplified classical noise in a fiber Raman generator," Opt. Express, vol. 13, pp. 2948-2960, 2005.

[19] F. Vanholsbeeck, S. Martin-Lopez, M. Gonzalez-Herraez, and S. Coen, "The role of pump incoherence in continuous-wvae supercontinuum generation," Opt. Express, vol. 13, pp. 6615-6625, 2005.

[20] B. Barviau, S. Randoux, and P. Suret, "Spectral broadening of a multimode continuous-wave optical field propagation in the normal dispersion regime of a fiber," Opt. Lett., vol. 31, pp. 1696-1698, 2006.

[21] R. Osborne, "Raman pulse walk-off in single-mode fibers: An exact analysis," J. Opt. Soc. Amer. B, vol. 6, pp. 1726-1731, 1989.

[22] R. R. Alfano, Q. X. Li, T. Jimbo, J. T. Manassah, and P. P. Ho, "Induced spectral broadening of a wek picosecond pulse in glass produced by an intense picosecond pulse," Opt. Lett., vol. 11, pp. 626-628, 1986.

[23] C. Martinelli, L. Lorcy, A. Durécu-Legrand, D. A. Mongardien, and S. Borne, "Inlfuence of polarization on pump-signal RIN tranfer and cross-phase modulation in copumped Raman amplifiers," J. Lightw. Technol., vol. 24, pp. 3490-3505, 2006.

[24] B. E. Olsson, P. Öhlen, L. Rau, and D. J. Blumenthal, “A simple and robust $40-\mathrm{Gb} / \mathrm{s}$ wavelength converter using fiber cross-phase modulation and optical filtering," IEEE Photon. Technol. Lett., vol. 12, pp. 846-848, 2000.

[25] M. Galili, L. K. Oxenlowe, H. C. H. Mulvad, A. T. Clausen, and P. Jeppesen, "Optical wavelength conversion by cross-phase modulation of data signals up to $640 \mathrm{Gps,"} \mathrm{IEEE} \mathrm{J.} \mathrm{Sel.} \mathrm{Top.} \mathrm{Quantum} \mathrm{Electron.,}$ vol. 14, pp. 573-579, 2008.

[26] W. Wang, H. N. Poulsen, L. Rau, H.-F. Chou, J.-E. Bowers, and D. J. Blumenthal, "Raman-enhanced regenerative ultrafast all-optical fiber XPM wavelength converter," J. Lightw. Technol., vol. 23, pp. $1105-1115,2005$.

[27] L. Garcia, J. Jenkins, Y. Lee, N. Poole, K. Salit, P. Sidereas, C. G. Goedde, and J. R. Thompson, "Influence of classical pump noise on long-pulse multiorder stimulated Raman scattering in optical fiber," $J$. Opt. Soc. Am. B, vol. 19, pp. 2727-2736, 2002.

[28] E. Landahl, D. Baiocchi, and J. R. Thompson, "A simple analytic model for noise shaping by an optical fiber Raman generator," Opt. Commun., vol. 150, pp. 339-347, 1998.

[29] G. Ravet, A. A. Fotidai, and P. Mégret, "Spectral broadening in Raman fiber amplifier pumped by partially coherent wave," in CLEO Europe, Munich, 2007.
[30] J. T. Manassah, "Induced phase modulation by noisy fields," Opt. Lett., vol. 16, pp. 1379-1381, 1991.

[31] C. Finot, B. Kibler, L. Provost, and S. Wabnitz, "Beneficial impact of wave-breaking or coherent continuum formation in normally dispersive nonlinear fibers," J. Opt. Soc. Am. B, vol. 25, pp. 1938-1948, 2008.

[32] C. Finot, G. Millot, C. Billet, and J. M. Dudley, "Experimental generation of parabolic pulses via Raman amplification in optical fiber," Opt. Express, vol. 11, pp. 1547-1552, 2003.

[33] A. M. Weiner, J. P. Heritage, and R. H. Stolen, "Self-phase modulation and optical pulse compression influenced by stimulated Raman scattering in fibers," J. Opt. Soc. Am. B, vol. 5, pp. 364-372, 1988.

[34] M. D. Mermelstein, C. Headley, and J. C. Bouteillier, "RIN transfer analysis in pump depletion regime for Raman fibre amplifiers," Electron. Lett., vol. 38, pp. 403-404, 2002.

[35] D. Schadt and B. Jaskorzynska, "Frequency chirp and spectra due to self-phase modulation and stimulated Raman scattering influenced by pulse walk-off in optical fibers," J. Opt. Soc. Am. B, vol. 4, pp. 856-862, 1987.

[36] C. Finot, S. Pitois, and G. Millot, "Regenerative 40-Gb/s wavelength converter based on similariton generation," Opt. Lett., vol. 30, pp. 1776-1778, 2005.

[37] P. V. Mamyshev, "All-optical data regeneration based on self-phase modulation effect," in European Conference on Optical Communication, ECOC'98, Madrid, Spain, 1998, pp. 475-476.

[38] M. Matsumoto, "Performance analysis and comparison of optical 3R regenerators utilizing self-phase modulation in fibers," J. Lightw. Technol., vol. 22, pp. 1472-1482, 2004.

[39] L. Provost, C. Finot, K. Mukasa, P. Petropoulos, and D. J. Richardson, "Design scaling rules for 2R-Optical self-phase modulation-based regenerators 2R regeneration," Opt. Express, vol. 15, pp. 5100-5113, 2007.

[40] C. Kouloumentas, N. Pleros, D. Zakynthinos, D. Apostolopoulos, O. Zouraraki, A. Tzanakaki, H. Avramopoulos, and I. Tomkos, "Packet clock recovery using a bismuth oxide fiber-based optical power limiter," Opt. Express, vol. 15, pp. 9948-9953, 2007.

[41] M. Asobe, A. Hirano, Y. Miyamoto, K. Sato, K. Hagimoto, and Y. Yamabayashi, "Noise reduction of $20 \mathrm{Gbit} / \mathrm{s}$ pulse train using spectrally filtered optical solitons," Electron. Lett., vol. 34, pp. 1135-1136, 1988.

[42] S. Pitois, C. Finot, and L. Provost, "Asymptotic properties of incoherent waves propagating in an all-optical regenerators line," Opt. Lett., vol. 32, pp. 3263-3265, 2007.

[43] S. Pitois, C. Finot, L. Provost, and D. J. Richardson, "Generation of localized pulses from incoherent wave in optical fiber lines made of concatened Mamyshev regenerators," J. Opt. Soc. Am. B, vol. 25, pp. 1537-1547, 2008.

[44] C. Fludger, V. Handerek, and R. J. Mears, "Pump to signal RIN transfer in Raman fiber amplifiers," J. Lightw. Technol., vol. 19, pp. 1140-1148, 2001.

[45] X. Z. Sang, D. Dimitropoulos, B. Jalali, and Ö. Boyraz, "Influence of pump-to-signal RIN transfer on noise figure in silicon Raman amplifiers," IEEE Photon. Technol. Lett., vol. 20, pp. 2021-2023, 2008.

[46] K. Keita, P. Delaye, R. Frey, and G. Roosen, "Relative intensity noise transfer of large-bandwidth pump lasers in Raman fiber amplifiers," $J$. Opt. Soc. Am. B, vol. 23, pp. 2479-2485, 2006. 